\title{
Risk factors for respiratory failure in pneumococcal pneumonia: the importance of pneumococcal serotypes
}

\author{
Joaquín Burgos ${ }^{1}$, Manel Luján²,3, Maria Nieves Larrosa ${ }^{4}$, Dionísia Fontanals ${ }^{5}$, \\ Guadalupe Bermudo², Ana Maria Planes ${ }^{3}$, Mireia Puig ${ }^{1,6}$, Jordi Rello ${ }^{3,7}$, \\ Vicenç Falcó ${ }^{1}$ and Albert Pahissa ${ }^{1,6}$
}

\begin{abstract}
Affiliations: 'Dept of Infectious Diseases, Hospital Universitari Vall d'Hebron, Vall d'Hebron Research Institute (VHIR), Universitat Autònoma de Barcelona, Barcelona, ${ }^{2}$ Dept of Pneumology, Hospital de Sabadell, Corporació Sanitaria Parc Taulí, Universitat Autònoma de Barcelona, Barcelona, ${ }^{3} \mathrm{CIBER}$ Enfermedades Respiratorias (CIBERES), Barcelona, “Dept of Microbiology, Hospital Universitari Vall d'Hebron, Vall d'Hebron Research Institute, Universitat Autònoma de Barcelona, Barcelona, ${ }^{5}$ Dept of Microbiology, UDIAT, Hospital de Sabadell, Corporació Sanitaria Parc Taulí, Universitat Autònoma de Barcelona, Barcelona, 'Spanish Network for Research in Infectious Diseases (REIPI), Seville, and ${ }^{7}$ Dept of Critical Care, Hospital Universitari Vall d'Hebron, Vall d'Hebron Research Institute, Universitat Autònoma de Barcelona, Barcelona, Spain.
\end{abstract}

Correspondence: J. Burgos, Dept of Infectious Diseases, Hospital Universitari Vall d'Hebron, Universitat Autònoma de Barcelona, Passeig Vall d'Hebron 119-129, 08035 Barcelona, Spain. E-mail: jburgosđavhebron.net

ABSTRACT Pneumococcal serotypes are one of the main determinants of pneumococcal disease severity; however, data about their implication in respiratory failure are scarce.

We conducted an observational study of adults hospitalised with invasive pneumococcal pneumonia to describe the host- and pathogen-related factors associated with respiratory failure.

Of 1258 adults with invasive pneumococcal disease, 615 (48.9\%) had respiratory failure at presentation. Patients with respiratory failure were older $(62.1$ years versus 55.4 years, $\mathrm{p}<0.001)$ and had a greater proportion of comorbid conditions. They also had a greater proportion of septic shock $(41.7 \%$ versus $6.1 \%$, $\mathrm{p}<0.001)$, required admission to the intensive care unit more often $(38.4 \%$ versus $4.2 \%, \mathrm{p}<0.001)$ and had a higher mortality $(25.5 \%$ versus $3.5 \%, \mathrm{p}<0.001)$. After adjustment, independent risk factors for respiratory failure were: age $>50$ years (OR 1.63, 95\% CI 1.15-2.3), chronic lung disease (OR 1.54, 95\% CI 1.1-2.15), chronic heart disease (OR 1.49, 95\% CI 1.01-2.22) and infection caused by serotypes 3 (OR 1.97, 95\% CI 1.23-3.16), 19A (OR 2.34, 95\% CI 1.14-4.42) and 19F (OR 3.55, 95\% CI 1.22-10.28).

In conclusion, respiratory failure is a frequent complication of pneumococcal pneumonia and causes high morbidity and mortality. Pneumococcal serotypes 3, 19A and 19F are the main risk factors for this complication.

@ERSpublications

Respiratory failure in invasive pneumonia is determined by older age, comorbidities and serotypes 3, 19A and 19F http://ow.ly/qHN6D

For editorial comments see page 331 .

Earn CME accreditation by answering questions about this article. You will find these at the back of the printed copy of this issue or online at www.erj.ersjournals.com/site/misc/cmeinfo.xhtml

Received: March 202013 | Accepted after revision: June 282013 | First published online: July 112013

Support statement: This work was institutionally supported by the Spanish Network for Research in Infectious Diseases (REIPI), RD06/008 from the Ministry of Science and Innovation, "Instituto de Salud Carlos III" and Centro de Investigación Biomédica en Red (CIBER) de Enfermedades Respiratorias (grant number 06/06/36).

Conflict of interest: None declared.

Copyright @ERS 2014 


\section{Introduction}

Streptococcus pneumoniae is the leading cause of pneumonia worldwide. Despite the improvements in different medical aspects and critical care of patients, mortality remains unacceptably high [1]. Different factors related to the severity and mortality of pneumococcal disease have been described. Some of them are host-related, such as extremes of age, comorbidities or immunosuppressive conditions, and it is well known that they all are associated with an increased susceptibility to pneumococcal disease and also to a greater severity of the illness [2-4].

Nowadays there is increasing evidence that organism-related factors also play a key role in the clinical course of the disease. The capsular polysaccharide is probably the major virulence determinant of S. pneumoniae, protecting it from phagocytosis [5]. At present, more than 90 pneumococcal serotypes have been described on the basis of differences in the antigenic characteristics of capsular polysaccharides. Experimental studies in animal models have shown that pneumococcal serotypes differ in properties such as resistance to phagocytosis, ability to penetrate into tissues and capacity to activate the inflammatory response $[5,6]$. Different studies have explored the relationship between serotypes and clinical presentation of pneumococcal disease, and associations with the severity of the illness and mortality have been demonstrated $[4,7-10]$.

The clinical spectrum of pneumococcal pneumonia is wide, from an almost asymptomatic disease in some cases to a devastating illness complicated with respiratory failure and septic shock in others. Septic shock is one of the most important factors influencing prognosis of patients with pneumococcal pneumonia. Interestingly, recent reports have suggested that infection caused by serotypes 3 and 19A are independent risk factors for this complication [10,11]. Respiratory failure is also an important and severe complication of pneumococcal pneumonia that causes high morbidity and mortality. However, few data are available about the host- and pathogen-related factors associated with this complication. We hypothesise that specific serotypes could also play a role in the development of respiratory failure in patients with pneumococcal pneumonia.

The aim of our study was to analyse possible risk factors associated with the development of respiratory failure in adult patients with invasive pneumococcal pneumonia (IPP). We also aimed to investigate the role of any specific serotype in this severe complication.

\section{Materials and methods}

Study population and setting

Patients were enrolled as part of an ongoing observational study initiated in 1996 of all adults (aged $\geqslant 18$ years) hospitalised with IPP in two teaching hospitals from Catalonia, Spain (Hospital Universitari Vall d'Hebron and Hospital Universitari Parc Tauli). In both hospitals, all microbiological strains isolated in sterile samples are collected systematically. When $S$. pneumoniae was isolated from a sterile sample, the patient was included in the study and all the clinical and evolutive data were collected prospectively. The study was approved by the ethics board of the participating centres. The need for informed consent was waived due to the observational nature of the study.

\section{Study variables and data collection}

From each patient, the following variables were recorded. 1) Sociodemographic and temporal data (age, sex, period of influenza epidemic, current tobacco smoking, long-term alcohol abuse and vaccination status with the 23-valent polysaccharide vaccine (PPV-23)); 2) underlying diseases (chronic lung disease, chronic heart disease, liver disease, cerebrovascular disease, diabetes and renal insufficiency); 3) immunosuppressive conditions (HIV infection, haematological cancer, solid cancer, solid organ or stem cell transplantation and current immunosuppressive therapy); 4) variables related to respiratory status (respiratory failure, need for mechanical ventilation and chest radiograph pattern); 5) other variables related to clinical presentation and outcome (septic shock, intensive care unit (ICU) admission, suppurative lung complications, length of hospital stay, mortality and pneumonia severity index);6) antimicrobial therapy; and 7) microbiological data (serotype and antibiotic susceptibility).

\section{Definitions}

IPP was diagnosed when a patient had consistent clinical findings plus a new pulmonary infiltrate on chest radiography and isolation of $S$. pneumoniae in blood and/or pleural fluid cultures [10]. Respiratory failure was defined as oxygen saturation of $<90 \%$ on room air or arterial oxygen tension to inspiratory oxygen fraction ratio $<250$ [12]. Mechanical ventilation was defined as any period of mechanically assisted ventilation via an endotracheal or nasotracheal tube. The radiographic examinations were performed by radiologists as routine examinations, and the extent of infiltrates was classified as lobar, bilobar or 
multilobar and uni- or bilateral. For the purpose of this study, we analysed the presence of these variables at the moment of presentation in the emergency department.

An episode was considered to be within the epidemic period if it was diagnosed during the weeks of the influenza epidemic each year. The influenza epidemic was defined as a weekly incidence of $>100$ cases per 100000 inhabitants in Catalonia, and information was obtained from the Catalan Public Health System [13].

Smoking status was considered when a patient had smoked $>10$ cigarettes per day for $\geqslant 1$ year, and alcoholism when a patient had consumed $>80 \mathrm{~g}$ of alcohol daily for $\geqslant 1$ year preceding the study. Chronic lung disease was defined on the basis of clinical, radiological and/or functional tests and included chronic obstructive pulmonary disease, severe asthma and interstitial lung disease. Septic shock was considered when vasoactive drugs were necessary to obtain appropriate arterial pressure values after fluid replacement. A patient was considered to be vaccinated if PPV-23 had ever been administered before admission, according to hospital and primary healthcare centre records.

\section{Microbiological procedures}

S. pneumoniae strains were identified by Gram staining, optochin susceptibility testing, bile solubility testing and latex agglutination testing. Antimicrobial susceptibility was determinated using the microdilution method in accordance with Clinical and Laboratory Standards Institute procedures [14]. For the purpose of this study, we classified pneumococcal isolates according the nonmeningitis breakpoints: penicillinsusceptible (minimum inhibitory concentration (MIC) $\leqslant 2 \mu \mathrm{g} \cdot \mathrm{mL}^{-1}$ ) or cefotaxime-susceptible (MIC $\left.\leqslant 1 \mu \mathrm{g} \cdot \mathrm{mL}^{-1}\right)$. Serotypes were performed by Quellung reaction and/or dot-blot assay at the Spanish Reference Laboratory for Pneumococci (Instituto de Salud Carlos III, Majadahonda, Spain).

\section{Statistical analysis}

We estimated attributable risk of respiratory failure due to any specific serotype as the difference in rate between the specific serotype and serotype 8 . We chose this serotype as the reference group because it represents a frequent serotype in our study and had a similar distribution in the risk of respiratory failure in the univariate analysis between the two groups $(\mathrm{OR} \sim 1)$.

To identify the risk factors for respiratory failure, need for mechanical ventilation and chest radiograph pattern were compared as dichotomous variables using a Chi-squared test, and continuous variables using a ttest. To exclude variables with high co-linearity from the multivariate analysis, those significantly associated in the univariate analysis were assessed for bivariate correlation. We excluded significantly correlated variables $(\mathrm{p}<0.001)$ from further analysis. Clinically significant variables and those with $\mathrm{p}<0.1$ in the univariate analysis were entered as covariates into a forward stepwise logistic regression analysis for respiratory failure, need for mechanical ventilation and chest radiograph pattern. Odds ratios with $95 \%$ confidence intervals are reported. The fit of the model was tested using the Hosmer-Lemeshow goodness-of-fit test. Analyses were conducted using the statistical software package SPSS (version 15.0; IBM, Armonk, NY, USA).

\section{Results}

\section{Patient characteristics}

Over the study period, 1258 consecutive adults with IPP were diagnosed: 615 (48.9\%) of them had respiratory failure at admission. Clinical features and demographic characteristics of the patients are shown in table 1.

Patients with respiratory failure were significantly older and had a greater proportion of comorbid conditions. Patients were also more likely to have received prior pneumococcal vaccine than patients without respiratory failure $(24.1 \%$ versus $17.1 \%, \mathrm{p}=0.015)$. Regarding the severity of illness, patients with respiratory failure were more likely to have a bilateral pneumonia with multilobar involvement. As expected, they more frequently needed mechanical ventilation. They also had a greater proportion of septic shock and required ICU admission more often than patients without respiratory failure. The case fatality rate was significantly greater $(25.5 \%$ versus $3.5 \%, \mathrm{p}<0.001)$. In contrast, the proportion of suppurative complications was similar in both groups.

\section{Serotype distribution and antibiotic susceptibility}

Overall, 1029 (81.8\%) pneumococcal strains were available for serotyping. The most frequent serotypes are shown in figure 1. Pneumococcal serotypes most commonly associated with respiratory failure were serotypes 3, 19A and 19F (respiratory failure developed in $65.8 \%$ of infections by serotype 3, in $65.5 \%$ by serotype $19 \mathrm{~A}$ and in $81.5 \%$ by serotype $19 \mathrm{~F}$ ). The excess risks of respiratory failure attributable to each were 20.4\% (95\% CI 4.6-35.1\%) for serotype 3, 20.1\% (95\% CI 1.7-36.6\%) for serotype 19A and 36\% (95\% CI $16.3-55.7 \%)$ for serotype 19F. Serotypes 3 and 19A were the most frequently isolated in patients with 
TABLE 1 Basal characteristics, clinical presentation and microbiological data of pneumococcal pneumonia patients

Presenting with respiratory failure Presenting without respiratory failure

p-value

\begin{tabular}{lc} 
Subjects $n$ & 615 \\
Sociodemographic and temporal variables & 62.13 \\
Age years & $411 / 615(66.9)$ \\
Male & $155 / 513(25.2)$ \\
Period of influenza epidemic & $124 / 513(24.1)$ \\
Previous pneumococcal vaccination & $341 / 607(56.2)$ \\
Smoking & $127 / 605(21)$ \\
Heavy alcohol consumption & \\
Underlying disease & $216 / 607(35.6)$ \\
Chronic lung disease & $140 / 605(23.1)$ \\
Chronic heart disease & $75 / 605(12.4)$ \\
Cerebrovascular disease & $94 / 611(15.4)$ \\
HIV infection & $46 / 610(7.5)$ \\
Haematological cancer & $84 / 610(13.8)$ \\
Solid cancer & \\
Clinical presentation and outcomes & $210 / 614(34.2)$ \\
Bilateral pneumonia & $347 / 614(58.2)$ \\
Bilobar or multilobar pneumonia & $254 / 609(41.7)$ \\
Septic shock & $234 / 609(38.4)$ \\
ICU admission & $533 / 609(87.6)$ \\
PSI $4^{\#}$ & $161 / 609(26.1)$ \\
Mechanical ventilation & 17.83 \\
Length of hospital stay days & $120 / 615(19.5)$ \\
Empyema & $157 / 615(25.5)$ \\
Hospital mortality & \\
Microbiological data & $42 / 480(8.8)$ \\
Serotype 1 & $79 / 480(16.5)$ \\
Serotype 3 & $27 / 480(5.6)$ \\
Serotype 7F & $25 / 480(5.2)$ \\
Serotype 8 & $38 / 480(7.9)$ \\
Serotype 19A & $22 / 480(4.6)$ \\
Serotype 19F & $2 / 480(0.4)$ \\
Serotype 23A & $5 / 480(1)$ \\
Serotype 23F & $600 / 615(97.5)$ \\
Penicillin susceptibility & $601 / 615(97.7)$ \\
Cephalosporin susceptibility ${ }^{+}$ & \\
\hline
\end{tabular}

\begin{tabular}{|c|c|}
\hline 55.42 & $<0.001$ \\
\hline $388 / 615(60.4)$ & 0.019 \\
\hline $169 / 643(26.3)$ & NS \\
\hline $96 / 561(17.1)$ & 0.015 \\
\hline $349 / 631$ (55.3) & NS \\
\hline $101 / 629(16.1)$ & 0.028 \\
\hline $144 / 631(22.8)$ & $<0.001$ \\
\hline $76 / 623(12.2)$ & $<0.001$ \\
\hline $40 / 623(6.4)$ & 0.001 \\
\hline $124 / 638(19.4)$ & 0.063 \\
\hline 47/638 (7.4) & NS \\
\hline $68 / 638(10.3)$ & 0.068 \\
\hline $63 / 643$ (9.8) & $<0.001$ \\
\hline $109 / 643$ (17) & $<0.001$ \\
\hline $39 / 638(6.1)$ & $<0.001$ \\
\hline $27 / 638$ (4.2) & $<0.001$ \\
\hline $328 / 638(51.4)$ & $<0.001$ \\
\hline $15 / 639(2.3)$ & $<0.001$ \\
\hline 10.62 & $<0.001$ \\
\hline $120 / 643$ (18.7) & NS \\
\hline $23 / 643$ (3.5) & $<0.001$ \\
\hline $98 / 543$ (18) & $<0.001$ \\
\hline $41 / 523(7.6)$ & $<0.001$ \\
\hline $45 / 523$ (8.3) & 0.111 \\
\hline $30 / 523(5.4)$ & 0.467 \\
\hline $20 / 523$ (3.7) & 0.004 \\
\hline $5 / 523$ (0.9) & $<0.001$ \\
\hline $9 / 523$ (1.7) & 0.07 \\
\hline $16 / 523(2.9)$ & 0.045 \\
\hline $633 / 643(98.5)$ & NS \\
\hline $624 / 643$ (97) & NS \\
\hline
\end{tabular}

Data are presented as mean or $\mathrm{n} / \mathrm{N}(\%)$, unless otherwise stated. ICU: intensive care unit; PSI: pneumonia severity index; NS: nonsignificant. ${ }^{\#}$ : PSI score $\geqslant 4$ at the moment of admission to the emergency department; ${ }^{9}$ : includes isolates with minimum inhibitory concentration $\left(\mathrm{MIC}^{\circ} \leqslant 2 \mu \mathrm{g} \cdot \mathrm{mL}^{-1}\right.$; ${ }^{+}$: includes isolates with MIC $\leqslant 1 \mu \mathrm{g} \cdot \mathrm{mL}^{-1}$.

bilateral infiltrates and in those who needed of mechanical ventilation. In contrast, serotype 1 was less frequently isolated in cases of respiratory failure with an attributable risk of $-15.4 \%$ (95\% CI -30.6- $-0.6 \%$ ). Regarding antibiotic susceptibility, no differences in the rate of resistance to penicillin and cephalosporin were found between both groups.

\section{Multivariate analysis of risk factors}

The results of the multivariate analysis are shown in tables 2-4. After adjustment by age, comorbidities and pneumococcal vaccine status, independent risk factors for respiratory failure were: age $>50$ years (OR 1.63), chronic lung disease (OR 1.54), chronic heart disease (OR 1.49), and infection caused by serotype 3 (OR 1.97), serotype 19A (OR 2.34) and serotype 19F (OR 3.55) (Hosmer-Lemeshow $\mathrm{p}=0.96$ ). Pneumococcal pneumonia caused by serotype 1 showed a trend to be a protective factor. In the multivariate analysis, we did not find independent associations between respiratory failure and previous pneumococcal vaccination. Because patients with underlying chronic lung disease may have chronic respiratory insufficiency, we excluded these patients from the analysis in a second model, obtaining similar results.

To assess the impact of pneumococcal serotypes in young patients, we analysed the subgroup of patients aged $\leqslant 50$ years. After adjustment, the only independent factors associated with respiratory failure were 


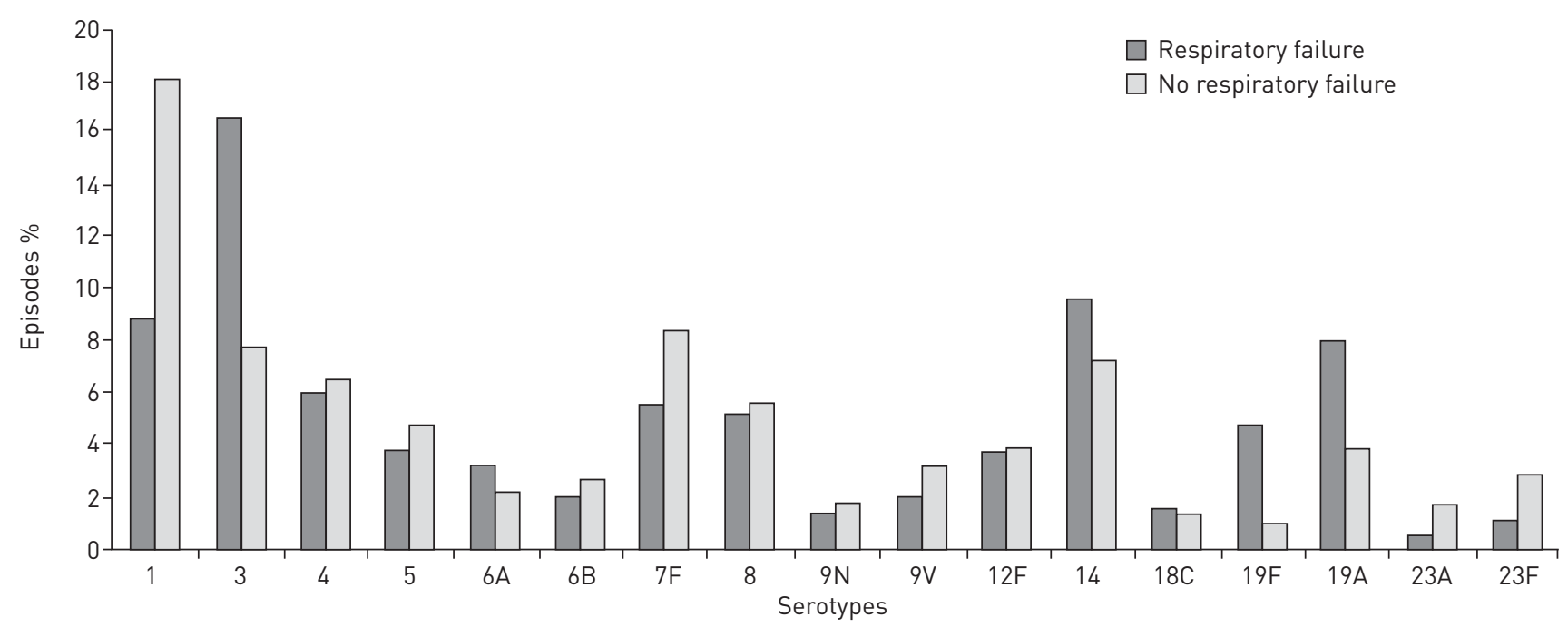

FIGURE 1 Serotypes causing invasive pneumococcal pneumonia in patients presenting with or without respiratory failure.

heavy alcohol consumption (OR 2.26) and infections caused by serotype 19A (OR 4.32) and serotype 19F (OR 9.82) (Hosmer-Lemeshow $\mathrm{p}=0.94)$.

Multivariate analysis also found that age $>50$ years (OR 1.5), serotype 3 (OR 2.69) and serotype 19A (OR 2.75) were independent risk factors for bilateral infiltrates in the radiological pattern (Hosmer-Lemeshow $\mathrm{p}=0.86$ ). In the same way, smoking (OR 1.99) and infections caused by serotype 3 (OR 2.97), serotype 19A (OR 2.43) and serotype 19F (OR 3.3) were identified as independent determinants of the need for mechanical ventilation (Hosmer-Lemeshow $\mathrm{p}=0.84$ ).

\section{Discussion}

The results of this large observational study of hospitalised adults with IPP support the hypothesis that specific pneumococcal serotypes play a key role in the development of respiratory failure in patients with pneumococcal pneumonia. This observation is of particular interest in the era of conjugate vaccines, because of the continuous replacement of serotypes. In our study, serotypes 3, 19A and 19F were found to be the main risk factors for respiratory failure, bilateral involvement upon radiography and need for mechanical ventilation.

Several studies have shown that specific serotypes are associated with different clinical patterns of pneumococcal disease. BRUEGgemann et al. [7] classified pneumococcal serotypes according to their capacity to cause invasive disease. Serotypes 1, 5 and 7F were classified as highly invasive serotypes, and they were associated with invasive disease in younger adults; nevertheless they caused infections with low mortality rates. In contrast, serotypes $3,19 \mathrm{~F}$ and $23 \mathrm{~F}$ had a low invasive potential, affecting older patients with comorbidities and causing higher case-fatality rates [7-10]. Specific serotypes have also been related with particular clinical presentations of pneumococcal disease. Thus, after the implementation of the 7 -valent conjugate vaccine, an increase of suppurative complications were observed, associated with the emergence of serotypes 1 and $3[15,16]$. In the same way, recent reports have found that septic shock in patients with pneumococcal pneumonia developed more frequently in infections caused by serotypes 3 and 19A $[10,11]$. However, to our knowledge, no previous studies have addressed the relationship between pneumococcal serotypes and respiratory failure. In our study, we found that serotypes 3, 19A and 19F were the main independent risk factors for development of respiratory failure, even over the risk related to age and comorbidities. These findings are consistent with previous data that associated these serotypes with greatest severity and mortality rates of pneumococcal disease $[17,18]$. Although not statistically significant, serotype 1 showed a trend to be a protective factor of the development of respiratory failure. The paradox of this serotype with a high capacity to develop invasive illness and empyema in young adults but with a lesser tendency to cause severe illness $[6,15,16]$ could explain why the rates of empyema were similar in both groups.

The pathogenic mechanism that would explain why some specific pneumococcal serotypes might cause respiratory failure is unknown. It has been suggested that heavily encapsulated serotypes could better resist neutrophil-mediated killing [19] and induce a greater inflammatory response causing a more severe disease [17]. In fact, serotypes 3, 19F and 19A, which we identified as the main risk factors for respiratory failure, match those that have been found to be heavily encapsulated [17]. However, it is certain that other 


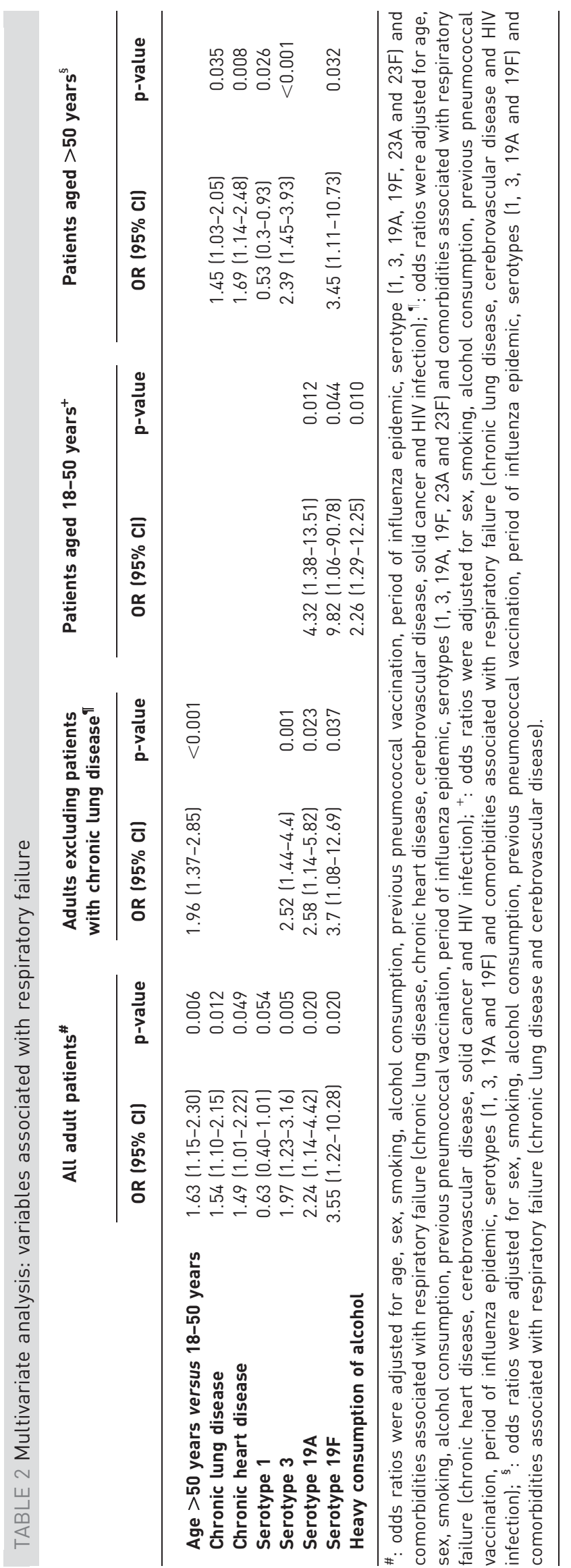


TABLE 3 Multivariate analysis: variables associated with bilateral infiltrates

\begin{tabular}{lcr} 
& OR $(\mathbf{9 5 \%} \mathbf{C l})$ & p-value \\
\hline Age $>$ 50 years versus 18-50 years & $1.5(1.07-2.1)$ & 0.018 \\
Serotype 3 & $2.69(1.74-4.14)$ & $<0.001$ \\
Serotype 19A & $2.75(1.55-4.88)$ & 0.001 \\
\hline
\end{tabular}

Odds ratios were adjusted for sex, smoking, alcohol consumption, previous pneumococcal vaccination, period of influenza epidemic, serotypes (1,3,12,19A and 19F) and comorbidities associated with bilateral infiltrates (solid cancer and HIV infection).

serotypes with a thick capsule, such as serotype 8, are not associated with a greater risk of respiratory failure, so other factors should be involved.

Recently, SANCHEZ et al. [20] found that serotypes differ in their ability to adhere to the respiratory epithelium due to the expression of different adhesins, having an impact on virulence. We hypothesise that the pneumococcus requires both factors, bacterial attachment and resistance to phagocytosis, in order to persist and prevent bacterial clearance from the alveoli. This phenomenon could facilitate an excessive inflammatory response that finally leads to cellular damage and lung injury.

Other factors of the pneumococci, rather than capsular polysaccharides, are also implicated in their virulence and may affect disease severity. In this way different studies reveal an association between the amount of bacterial load of pneumococci and the risk of developing septic shock, the need for mechanical ventilation and the mortality of pneumococcal disease [21, 22]. The genetic properties of pneumococci might also play a role. The relationship between these factors and the pathogenic basis for these observations is complex and poorly understood.

In addition to pathogen related factors, host aspects also have a significant role in the severity of the illness [2-4]. As in our study, other authors reported that older age and chronic lung disease were independent risk factors for respiratory failure $[23,24]$. Interestingly, it should be noted that the serotypes, and not the underlying disease, were the main risk factors for respiratory failure in the multivariate analysis. This is best exemplified in the group of young adults without comorbidities in whom the causal serotype became the determining factor of outcome. To avoid confusion regarding the role of underlying chronic lung disease in the propensity to cause respiratory failure, we performed an analysis excluding these patients, obtaining similar results.

Nevertheless, we must keep in mind that this study did not evaluate other host factors that might also influence the risk of developing respiratory failure. Recently, specific genetic polymorphisms have been associated with a poor respiratory outcome in pneumococcal pneumonia [23,25]. Therefore, a tendency to a particular presentation of pneumonia might also be genetically determined for the ability of the individual to respond to the infection.

There is also some debate about the beneficial effects of the prior administration of the PPV-23 improving clinical outcomes in patients with pneumonia. Some studies have observed better clinical outcomes in patients who had previously received the PPV-23 compared with unvaccinated individuals [26, 27]. We also observed this beneficial effect on the incidence of septic shock and survival in vaccinated patients with HIV infection who developed an invasive pneumococcal infection [28]. Regarding respiratory outcomes, FISMAN

TABLE 4 Multivariate analysis: variables associated with mechanical ventilation

\begin{tabular}{lcr} 
& OR $(95 \%$ CI) & p-value \\
\hline Smoking & $1.99(1.34-2.94)$ & 0.001 \\
Solid cancer & $0.42(0.2-0.87)$ & 0.019 \\
Serotype 3 & $2.97(1.84-4.8)$ & $<0.001$ \\
Serotype 19A & $2.43(1.25-4.72)$ & 0.001 \\
Serotype 19F & $3.3(1.32-8.21)$ & 0.010 \\
\hline
\end{tabular}

Odds ratios were adjusted for sex, smoking, alcohol consumption, previous pneumococcal vaccination, period of influenza epidemic, serotypes $(1,3,19 \mathrm{~A}, 19 \mathrm{~F}$ and $23 \mathrm{~F})$ and comorbidities associated with mechanical ventilation (solid cancer). 
et al. [27] found that prior receipt of pneumococcal vaccine was associated with a decreased risk of respiratory failure in a large cohort of adults with pneumonia. In our experience we have not been able to find a benefit of PPV-23 in the risk of respiratory failure. It is possible that the relatively low rates of vaccination, the limited number of patients and the confounding factors derived from the fact that patients with increased risk of respiratory complications have a stronger indication to receive PPV-23, make it difficult to establish this association.

It is known that influenza plays an important role in the incidence of pneumococcal pneumonia [29]. Moreover, animal models suggest that previous influenza infection could result in a more severe pneumococcal disease [30]. Although we do not have information regarding this viral co-infection in each patient, we analysed the risk factors of respiratory failure according to the period of influenza epidemic. The results suggest that serotypes continue to be the main risk factor for respiratory failure, irrespective of the period of influenza infection.

Some limitations of our study must be pointed out. Firstly, the study included patients with IPP, who represent only a proportion of all patients with pneumococcal pneumonia, which may cause a possible bias due to the selection of the most invasive serotypes. This therefore makes it difficult to generalise the findings to all cases of pneumococcal pneumonia. It is noteworthy that the majority of studies focused in pneumococcal serotypes are performed in patients with invasive disease. Secondly, our study presents data from only two centres, so the results might not translate to other geographical areas where specific serotype distribution could differ. Finally, and despite the adjustment for an important number of covariables, other potential factors that might modulate the clinical presentation of pneumococcal pneumonia such as genetic properties of S. pneumoniae strains, genetic characteristics of the host or the effect of other viral coinfections have not been evaluated in our study.

Despite these limitations, we believe that our study has shown important and novel findings about the clinical presentation of invasive pneumococcal pneumonia. We have identified pneumococcal serotypes 3 , $19 \mathrm{~A}$ and $19 \mathrm{~F}$ as the main determinants of respiratory failure, extensive radiograph involvement and need for mechanical ventilation, after adjustment by age, comorbidities and pneumococcal vaccination. This observation is of particular interest in the era of conjugate vaccines, because of the continuous replacement of serotypes.

\section{References}

Varon E, Mainardi JL, Gutmann L. Streptococcus pneumoniae: still a major pathogen. Clin Microbiol Infect 2010; 16: 401.

2 Lipsky BA, Boyko EJ, Inui TS, et al. Risk factors for acquiring pneumococcal infections. Arch Intern Med 1986; 146: 2179-2185.

3 Musher DM. Infections caused by Streptococcus pneumoniae: clinical spectrum, pathogenesis, immunity, and treatment. Clin Infect Dis 1992; 14: 801-809.

4 Alanee SR, McGee L, Jackson D, et al. Association of serotypes of Streptococcus pneumoniae with disease severity and outcome in adults: an international study. Clin Infect Dis 2007; 45: 46-51.

5 Guckian JC, Christensen GD, Fine DP. The role of opsonins in recovery from experimental pneumococcal pneumonia. J Infect Dis 1980; 142: 175-190.

6 Engerlhad D, Pomeranz S, Gallily R, et al. Serotype-related differences in inflammatory response to Streptococcus pneumoniae in experimental meningitis. J Infect Dis 1997; 175: 979-982.

7 Brueggemann AB, Peto TE, Crook DW, et al. Temporal and geographic stability of the serogroup-specific invasive disease potential of Streptococcus pneumoniae in children. J Infect Dis 2004; 190: 1203-1211.

8 Sjöström K, Spindler C, Ortqvist A, et al. Clonal and capsular types decide whether pneumococci will act as a primary or opportunistic pathogen. Clin Infect Dis 2006; 42: 451-459.

9 Jansen AG, Rodenburg GD, van der Ende A, et al. Invasive pneumococcal disease among adults: associations among serotypes, disease characteristics, and outcome. Clin Infect Dis 2009; 49: e23-e29.

10 Burgos J, Falcó V, Borrego A, et al. Impact of the emergence of non-vaccine pneumococcal serotypes on the clinical presentation and outcome of adults with invasive pneumococcal pneumonia. Clin Microbiol Infect 2013; 19: 385-391.

11 Garcia-Vidal C, Ardanuy C, Tubau F, et al. Pneumococcal pneumonia presenting with septic shock: host- and pathogen-related factors and outcomes. Thorax 2010; 65: 77-81.

12 Azoulay E, Mokart D, Lambert J, et al. Diagnostic strategy for hematology and oncology patients with acute respiratory failure: randomized controlled trial. Am J Respir Crit Care Med 2010; 182: 1038-1046.

13 PIDIRAC. Canal Salut. www20.gencat.cat/portal/site/canalsalut/menuitem.41e04b39494flbe3ba963bb4b0c0e1a0/ ?vgnextoid = db0d438bd384c310VgnVCM2000009b0c1e0aRCRD\&vgnextchannel=db0d438bd384c310VgnVCM 2000009b0cle0aRCRD\&vgnextfmt=default Date last updated: June 25, 2013. Date last accessed: February 28, 2013.

14 Clinical and Laboratory Standard Institute (CLSI). Performance Standards for Antimicrobial Susceptibility Testing; Twenty-Second Informational Supplement. CLSI document M100-S22. Wayne, Clinical and Laboratory Standards Institute, 2012.

15 Byington CL, Korgenski K, Daly J, et al. Impact of the pneumococcal conjugate vaccine on pneumococcal parapneumonic empyema. Pediatr Infect Dis J 2006; 25: 250-254. 
16 Burgos J, Lujan M, Falcó V, et al. The spectrum of pneumococcal empyema in adults in the early 21 st century. Clin Infect Dis 2011; 53: 254-261.

17 Weinberger DM, Harboe ZB, Sanders EA, et al. Association of serotype with risk of death due to pneumococcal pneumonia: a meta-analysis. Clin Infect Dis 2010; 51: 692-699.

18 Harboe ZB, Thomsen RW, Riis A, et al. Pneumococcal serotypes and mortality following invasive pneumococcal disease: a population-based cohort study. PLoS Med 2009; 6: e1000081.

19 Weinberger DM, Trzciński K, Lu YJ, et al. Pneumococcal capsular polysaccharide structure predicts serotype prevalence. PLoS Pathog 2009; 5: e1000476.

20 Sanchez CJ, Hinojosa CA, Shivshankar P, et al. Changes in capsular serotype alter the surface exposure of pneumococcal adhesins and impact virulence. PLoS One 2011; 6: e26587.

21 Rello J, Lisboa T, Lujan M, et al. Severity of pneumococcal pneumonia associated with genomic bacterial load. Chest 2009; 136: 832-840.

22 Werno AM, Anderson TP, Murdoch DR. Association between pneumococcal load and disease severity in adults with pneumonia. J Med Microbiol 2012; 61: 1129-1135.

23 Waterer GW, Quasney MW, Cantor RM, et al. Septic shock and respiratory failure in community-acquired pneumonia have different TNF polymorphism associations. Am J Respir Crit Care Med 2001; 163: 1599-1604.

24 Marfin A, Sporrer J, Moore P, et al. Risk factors for adverse outcome in persons with pneumococcal pneumonia. Chest 1995; 107: 457-462.

25 Quasney MW, Waterer GW, Dahmer MK, et al. Association between surfactant protein B + 1580 polymorphism and the risk of respiratory failure in adults with community-acquired pneumonia. Crit Care Med 2004; 32: $1115-1119$.

26 Johnstone J, Marrie TJ, Eurich DT, et al. Effect of pneumococcal vaccination in hospitalized adults with community-acquired pneumonia. Arch Intern Med 2007; 167: 1938-1943.

27 Fisman DN, Abrutyn E, Spaude KA, et al. Prior pneumococcal vaccination is associated with reduced death, complications, and length of stay among hospitalized adults with community-acquired pneumonia. Clin Infect Dis 2006; 42: 1093-1101.

28 Imaz A, Falcó V, Peñaranda M, et al. Impact of prior pneumococcal vaccination on clinical outcomes in HIVinfected adult patients hospitalized with invasive pneumococcal disease. HIV Med 2009; 10: 356-363.

29 Grijalva G, Griffin M. Unveiling the burden of influenza-associated pneumococcal pneumonia. J Infect Dis 2012; 205: 355-357.

30 McCullers JA. Insights into the interaction between influenza virus and pneumococcus. Clin Microbiol Rev 2006; 19: $571-582$. 